\title{
The research and design of user interface in parallel computer system
}

\author{
Liu Xiang $^{1}$ Shang Liyuan ${ }^{2}$ Lu Zhenting ${ }^{3}$ \\ 1. Department of Information Engineering of Jiangxi Technical College of Manufacturing, JiangXi \\ Nanchang, 330095; \\ 2. Department of Economics and Management of Jiangxi Technical College of Manufacturing, \\ JiangXi Nanchang, 330095; \\ 3. Electronic and Information Engineering Branch of Jiangxi Industry Polytechnic College, JiangXi \\ Nanchang,330095
}

Keywords: User interface; Parallel computer system; System design; Computer System

\begin{abstract}
The user interface is a broad concept, which exists in various fields of science and technology, measuring instrument dial, TV remote controller, and the radio knob, etc, all belonging to the category of user interface. With the emergence and development of the computer, it is used in every field of society and it is expanding rapidly. Therefore, the research of computer user interface becomes an important topic. It is convenient for the user to use the system, simplifies many complicated operation and instruction, draws designers and users closer, and has extremely important significance on the development and application of computer. It is of far-reaching significance to provide a description of the system to explain profound theories in simple language and coach user interface for the manufacturer or the user step by step. Study the developmental trend of the user interface, understand the parallel computer system user interface at home and abroad, analyze users' needs, and establish the design idea and model. Design user interface of parallel systems for massive parallel computing system and provide its interface with a variety of programming environment. It can be utilized in a certain range and feedback can be collected to prepare for future research.
\end{abstract}

\section{Introduction}

At present, there is not even a parallel computer system equipped with a sophisticated user interface both at home and abroad, which is regret. Most researchers who use parallel computing system slowly enter the operating system and programming with the help of handbook and user support staff. This is a waste of time and manpower. It also causes the negative attitude of the people towards the parallel machine as well as a waste of the high performance computer resources. Some of the user interface introducing parallel technology can only realize the textbook effect, but are unable to really help users familiar with the entire parallel system, and its programming and operation.

What runs in the parallel machine is parallel program, which is different from the serial program on a single computer in the daily use. Besides, parallel machine is far greater than the single one in structure and complexity of operation. Therefore, how to better introduce parallel systems and parallel processing technology to allow users to master the programming method more quickly and to use the system more conveniently has become an important topic for promotion of parallel computer. The solution to his topic depends on an intuitive, simple, but inclusive, extensible user interface to a great extent. 
The rapid development of science and technology requires a high performance computer to meet the needs of research. Massive parallel computer system fulfills the requirements. Due to different users, different cultural levels, different familiarity with the system and different purposes of using them, they will encounter a variety of problems when encountering the massive parallel computer system. From the development process of computer user interface, it walks on a graphical and networked road.

\section{The development of parallel machine}

With the popularization and development of computer technology, the promotion of network and increase of computer users, the user interface is getting more and more attention and the fields are in rapid expansion. In order to better meet the needs of users and enable some users to grasp the system faster, it is necessary to design a simple, friendly and extensible user interface, which is also one of the important standards to measure whether a software system is excellent.

Before the computer appeared, the user interface has come into being. Of course, here the interface refers to the general user interface. Take radio for example. The radio broadcasts on different frequency bands, and each radio can also receive all the signals in the corresponding frequency. Therefore, the radio knob and the number on the frequency bands are simple user interfaces. Then have a look at TV remote controller. Because each audience has a different choice of channels, voice, brightness and contrast, TV is designed to adjust the corresponding circuit to meet these needs. But for the viewer, the few small buttons on the remote controller can help him to complete the above selection. Obviously, TV remote controller is an example of user interface. The so-called user interface is a tool to help people with a simple, intuitive way to complete the operation.

The emergence of the computer plays a tremendous role in promoting the development of society. Early use of computers was machine-oriented language. Therefore, only professional programmers can use it. With the rapid development of computer hardware, computer software has also developed correspondingly. The complexity of computer systems is increasing and computer applications are expanding, which prompts the computer software to be richer and richer and more effective to utilize its hardware resources.

\section{HTML}

Text identifiable language (HTML) is used to produce a simple identification system of hypertext documents. The file generated by it is easy to be transplanted from one platform to another. In fact, the HTML file is the general semantic SGML file that can properly describe the application information in numerous fields. The HTML identification can describe the hypertext information, e-mail, documents and multimedia, options menu, database query results, files containing graphics with simple structure, hypertext view of the existing information body, etc.

WWW was originally intended to connect information from around the world. In order to achieve this, WWW uses hypertext transfer protocol that permits the transmission between the client and the server to complete the agreement, whose results are returned in the part MIME. HTML is one of the expressions used by WWW and is used as a MIME content type. In addition, HTML is also the application of generalized description language in line with international standard 1508879 which is used to define structured file types 
In the HTML file, additional tabs are used to define headings, paragraphs, lists, flashing characters and links. In the three layers of different specification, it provides different features, able to design the interface more flexibly and more conveniently to meet user's requirements. From 1992 to now, it has been widely used.

\section{The design of user interface}

From the user's point of view, the parallel computing system is difficult in application. Although it has powerful calculating ability, it has no friendly interface, which is very unfavorable to promote the dawn machine. Therefore, the design of parallel computing system interface is quite essential. It can shorten the distance between the user and parallel calculation and play the function of parallel machine better. Based on the above investigation and analysis, parallel computing system interface should be a multi-functional visual system integrating compiling, debugging, online help, process tracking and other functions so as to provide maximum operation and convenient for users.

In the large-scale project calculation, users often want to sit in his lab, access to relevant information on their own, while debugging and running the program, rather than run the program in the place of parallel machine. For general users, they want to understand the parallel computing system, and the most convenient way is through the network. Therefore, our user interface is based on Web browser. When the user inputs the network address of the parallel computing system, he can $\log$ into the parallel system, and work.

Based on the above analysis and design idea, two modes of the user interface model are designed in order for discussion and analysis.

Based on the HTML interface model, it is a simple interface with good consistency. But due to the use of Farme format, it is not intuitive and inconvenient for the user's query and the operation. The interface model, based on JAVA, is intuitive and closer to general window type design idea. It is user-friendly but the implementation is relatively complex. When involving the design and compiling in JAVA language, it also uses IGC to transfer text information to hypertext format to be browsed. It is not simple enough.

As the workstation cluster user interface, it is designed according to the characteristics of the single image. This characteristic is reflected in the following two aspects:

(1). The single entry point. Workstation cluster system externally provides a single name. Each machine in the cluster of workstations can have a different name. When the remote user wants to login to cluster system, he only needs to specify this single name, and the system will select a workstation with minimum load as the remote login entrance point. According to this characteristic, the user interface system will be loaded in each node, so, whichever node the system chooses as the entry point, it can provide the interface service to the user. At the same time, the user interface system should also display the state of each node for the system, enabling the user to understand his current location and meanwhile have a macro understanding of the whole station system.

(2). Single file system. Each node machine in the workstation cluster has its own file system, and there exists a single file system. All files in this system are placed on one node machine, but from every node machine in the workstation cluster can access to this file system. As with processing the local file, on every node machine, users can create, delete, read and write and set properties on the files in the single file system. According to this characteristic, the user interface system should provide the local file system and the single file system for respective process. If the remote user logs in from a certain node machine, he can only deal with files in this node and files in the single file 
system, but the files of other nodes cannot be processed. Of course, the interface should also provide the function of login to the other node, so that users can process different files on different node machine.

\section{Conclusion}

Parallel processing is a hot topic in the field of computer research. Large-scale parallel computer systems will gradually become the mainstream product of the current ultra high performance computer market. More and more scientific computing will also be carried out in parallel system. And its promotion, to a great extent, is related to whether there is a good user interface where the users at any level can get familiar with and use the system through a simple and friendly user interface. All sorts of factors put the research and development of parallel system user interface to an important position.

Based on KSIS interface, Dawning 100OPlus user interface is designed and developed. As for the workstation cluster method on the hardware in parallel computing system Plus system and the single systematic image on the software, the user interface is improved a lot, in order to better accord with the systematic characteristics. It is a good example of the scalability of the KslS interface. Actually the user interface of parallel computing system Plus itself is an instance of the extension of KsLs interface.

The real application shows that the user interface in parallel computing system and Plus system meet the needs of users and becomes a powerful assistant for them to access into the field of parallel programming. To research and develop user interface based on network in the large-scale parallel system is extremely challenging and creative, which is rather valuable both to users and researches. It facilitates the promotion and application of high-performance computer system in our country and sets up a bridge between the developers and users.

In conclusion, we have made our first step towards the new field of designing user interface in parallel system and have made some achievements and obtained valuable experience. I believe in the process of promoting and using large-scale parallel computer system, the user interface will be more and more mature and it will play a more important role.

\section{References}

[1] Said N M, Alias N, Ibrahim A, et al. Mathematical Model of Active Migration Glioma Growth on Parallel Computer System[J]. World Applied Sciences Journal, 2013, 23(12): 23-28.

[2] Painkras E, Plana L A, Garside J, et al. Spinnaker: a multi-core system-on-chip for massively-parallel neural net simulation[C]//Custom Integrated Circuits Conference (CICC), 2012 IEEE. IEEE, 2012: 1-4.

[3] Courtois P J. Decomposability: queueing and computer system applications[M]. Academic Press, 2014.

[4] Papakipos M N, Grant B K, Demetriou C G, et al. Systems and methods for compiling an application for a parallel-processing computer system: U.S. Patent 8,584,106[P]. 2013-11-12.

[5] Janssen C L, Adalsteinsson H, Cranford S, et al. A simulator for large-scale parallel computer architectures[J]. Technology Integration Advancements in Distributed Systems and Computing, 2012: 179. 
[6] Parallel Models of Associative Memory: Updated Edition[M]. Psychology Press, 2014.

[7] Barsness E L, Darrington D L, Peters A, et al. Managing database resources used for optimizing query execution on a parallel computer system: U.S. Patent 8,095,512[P]. 2012-1-10.

[8] Al Zain A D, Trinder P W, Hammond K. Orchestrating computational algebra components into a high-performance parallel system[J]. International Journal of High Performance Computing and Networking, 2012, 7(2): 76-86.

[9] Yousuf S E K, Mohammed S H A A. A parallel computer system for the detection and classification of breast masses[C]//Computing, Electrical and Electronics Engineering (ICCEEE), 2013 International Conference on. IEEE, 2013: 202-207.

[10]McGuire M S, Demetriou C G, Grant B K, et al. Application program interface of a parallel-processing computer system that supports multiple programming languages: U.S. Patent 8,745,603[P]. 2014-6-3. 